\title{
On-Chip, Mediator- and Membrane-Less Micro-Glucose Biofuel Cells Based on Layer-by-Layer Reduced Graphene Oxide-Enzyme Hybrids
}

\author{
Mengfan Wang, ${ }^{a, b}$ Tao Qian, ${ }^{a, b}$ Bo Liu, ${ }^{*, a, b}$ Chengchao Li, ${ }^{c}$ Libao Chen, ${ }^{c}$ and Chenglin Yan $^{*, a, b}$ \\ ${ }^{a}$ College of Physics, Optoelectronics and Energy \& Collaborative Innovation Center of Suzhou \\ Nano Science and Technology, Soochow University, Suzhou, Jiangsu 215006, China \\ ${ }^{b}$ Key Laboratory of Advanced Carbon Materials and Wearable Energy Technologies of \\ Jiangsu Province, Soochow University, Suzhou, Jiangsu 215006, China \\ ${ }^{c}$ State Key Laboratory of Powder Metallurgy, Central South University, Changsha, Hunan 410083, China
}

Email: jeje345@gmail.com (B. L.), c.yan@suda.edu.cn (C. Y.)

\begin{abstract}
Enzymatic biofuel cells (EBFCs) draw much attention since they use renewable fuels to generate electricity and power biological medical devices. However, such EBFCs' application as devices has always been a puzzle to researchers, let alone their microminiaturization. Herein, we show the efficient embedding of enzymes in a porous reduced graphene oxide (RGO) matrix to form a unique sandwich structure for constructing an on-chip glucose biofuel cell (GBFC). During electrochemical deposition, laccase and glucose oxidase are incorporated in RGO layers to be used as cathode and anode materials, respectively. This GBFC delivers a maximum volumetric power density of $14.77 \mathrm{~mW} \cdot \mathrm{cm}^{-3}$ under physiological conditions (50 mM glucose, $\mathrm{pH} 7$ ). Compared with previous work which is relatively large and cannot be separated from liquid phase system, our on-chip, mediatorand membrane-less micro-GBFC shows great potential to be applied as miniaturized devices to meet the rapidly growing need of being small, thin, wearable and even implantable in modern life.
\end{abstract}

Keywords on-chip energy devices, biofuel cells, layer-by-layer reduced graphene oxide, enzyme hybrids

\section{Introduction}

The recent rapid advance of biofuel cells $(\mathrm{BFCs})^{[1,2]}$ was stimulated by demands for environmentally-friendly, inexpensive, renewable catalysts and fuels. BFCs had a lot of advantages so as to be used as alternatives of conventional fuel cells and batteries. Differing from conventional fuel cells, which were catalyzed by noble materials, electricity of BFCs was mainly produced by the redox reactions of particular oxidizer and fuel at cathode and anode. Besides, BFCs can use renewable fuels such as lactate, ethanol, and sugar to generate electricity so as to be able to replace fossil fuels. Of all the biofuels, glucose stores high energy and it can be easily found in nature, while $\mathrm{O}_{2}$, as the oxidizer, is also freely available in daily life. Theoretically, since enzymatic catalysis possesses several satisfactory properties which can play a role at neutral $\mathrm{pH}$ and body temperature, BFCs can work indefinitely, thus making glucose biofuel cell (GBFC) wildly studied and great improvements being achieved in the last few years. ${ }^{[3-5]}$

At present, the limitation of GBFC lies in inefficient charge transfer between the electrodes and the enzymes. Therefore, it was highly desirable to realize direct electron transfer (DET) between the electrodes and redox active sites of enzymes to prevent the drawbacks of using mediators to connect with enzymes, such as leaching, voltage loss, and instability, thus maximizing the open circuit potential (OCP) of GBFC. ${ }^{[6,7]}$ However, GBFC that underwent DET was developed slowly due to the ease of creating mediated enzyme electrodes. ${ }^{[8-10]}$ Besides, achieving excellent catalytic currents by utilizing DET was not as simple as building a connection between electrode and enzyme. To date, sufficient loading of enzyme and active-site orientation remained to be the bottleneck of realizing an effective DET and one approach was explored to solve these problems: preparing a highly conductive matrix to immobilize the enzyme so as to ensure the occurrence of DET in any direction and sufficient current can be generated within DET distance.

Several carbon materials were utilized to connect the enzymes and the electrodes because of their high surface area and good conductivity. Cosnier and his co-workers ${ }^{[11]}$ fabricated a GBFC through efficiently wiring enzymes inside carbon nanotube matrix. Kim and his co-workers ${ }^{[12,13]}$ built the GBFC textiles from woven biscrolled carbon nanotube yarns with immobilized enzyme and redox mediator in it. Graphene and reduced graphene oxide (RGO) achieved great attention over the last few years and were applied in various fields due to their high surface area and special properties in mechanics, physics, and chemistry. ${ }^{[14,15]}$ To date, a handful of reports described the use of graphene or RGO as substrate for immobilization of enzyme.$^{[16,17]}$ In this case, RGO with excellent electrical conductivity, great electrochemical stability, and high surface area were considered as a desirable material to build DET between enzyme and electrode surface.

One of the most promising applications of biofuel cells was to power the implantable devices. These devices should not only utilize biofuels from the human body to generate power, but also be compatible with the human body, while not causing any inflammation. Biofuel cells stood out under such conditions, because most enzymes, as biocatalysts, reached their peak activity and catalyzed efficiently reactions at physiological temperatures and near-neutral $\mathrm{pH}$. Moreover, there was no requirement for separation of fuel and oxidant or separation of biofuel cell electrodes and physiological fluids because of en- 


\section{Report}

zymes' selectivity, thus allowing high mass-transfer rates and making the design of BFCs much simpler. However, such applications as devices were always a puzzle to researchers, let alone their microminiaturization. ${ }^{[18-20]}$ Nowadays, most biofuel cells still stayed in the stage of being tested in a simple double-electrode liquid phase system. It's still a tough challenge to turn them into microscale devices with high power output to meet the rapidly growing demands of smart fashion for being small, light, wearable, and even implantable.

In this work, we constructed an on-chip, mediator- and membrane-less micro-GBFC (Figure 1). 12 interdigital Au fingers (six fingers as anodes and cathodes, respectively) were deposited on a glass slide as substrate firstly. Bioanode and biocathode can be prepared by electrochemical reduction of graphene oxide to reduced graphene oxide in solutions of graphene oxide and enzymes. Through this electrochemical deposition method, a unique RGO-enzyme sandwich structure could be obtained. Such special layer-by-layer structure could effectively trap the enzymes and ensure high enzyme loading so that ion and electron transport could be sped up. Noteworthy, unlike physical methods, chemical constraining was able to maintain the activity of enzymes while not causing partial unfolding of proteins. Owing to the simplicity of the design, substantial miniaturization of the GBFC was possible, making the GBFC implantation less unachievable and thus allowing novel way for developing implantable and microscale device and solving the energy predicament.

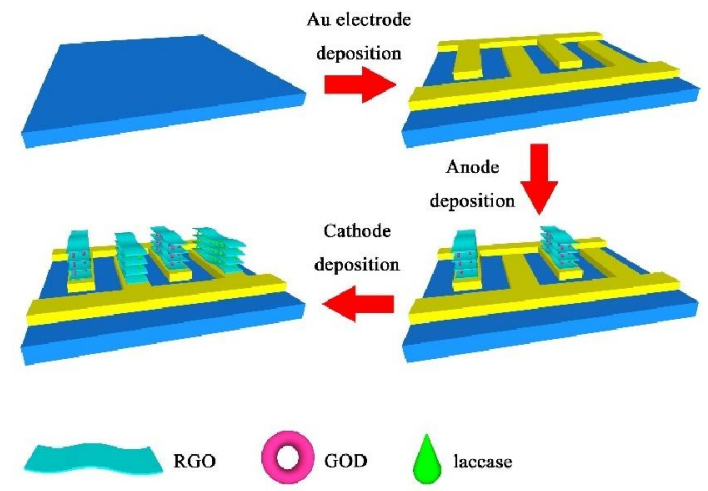

Figure 1 Fabricating process of on-chip, mediator- and membrane-less micro-glucose biofuel cell based on layer-bylayer reduced graphene oxide-enzyme hybrids.

\section{Experimental}

\section{Bioelectrode fabrication}

Bioanode was prepared by a simple electrochemical deposition approach. Briefly, graphene oxide (GO) was prepared from commercial graphite powder using modified Hummer's method. ${ }^{[21]} 30 \mathrm{mg}$ glucose oxidase (GOD) was added to $\mathrm{GO}$ dispersion $\left(3 \mathrm{mg} \cdot \mathrm{mL}^{-1}\right)$ and the resulting mixture was stirred for $0.5 \mathrm{~h}$. The substrate was placed in the solution and RGO was deposited on it through the reduction of GO by electrochemical method, in which process GOD was wrapped inside the RGO framework continuously at the same time. Finally, the chip was washed with deionized water and waited for biocathode deposition. The cathode was built in the same way by laccase instead of using GOD.

\section{Characterization}

The morphology was studied via a metallurgical microscope (Caikon Optical Instrument DMM-330C) and a field emission scanning electron microscope (FESEM, SU8010, Japan). The electrochemical deposition and electrochemical measurements were carried out in a conventional three-electrode system using the electrochemical workstation (CHI660E). The reference and counter electrodes were $\mathrm{Ag} / \mathrm{AgCl}$ and $\mathrm{Pt}$, respectively. They were placed in a glass container containing the phosphate buffer saline (PBS $0.1 \mathrm{M}, \mathrm{pH} 7,25^{\circ} \mathrm{C}$ ). For the complete GBFC cathode and anode were connected to the electrochemical workstation and the PBS electrolyte containing appropriate concentration of glucose was added to the electrode surface until PBS can totally cover the interdigital finger electrodes, and the performance of the GBFC was tested via two-electrode method.

\section{Results and Discussion}

As shown in the photograph of Figure $2 a$, a real GBFC based on Au electrode was consisted of 12 interdigital fingers, which were utilized as electrodes. Figure $2 \mathrm{~b}$ showed the schematic diagram of GBFC, in which the length and width of each finger were 4.8 and $0.1 \mathrm{~mm}$, and the distance between each electrode is $0.1 \mathrm{~mm}$. In Figure $3 a$, the bioanode was deposited firstly and the RGO-GOD hybrid adhered accurately to Au substrate. After the biocathode was deposited in Figure $3 b$ (a)

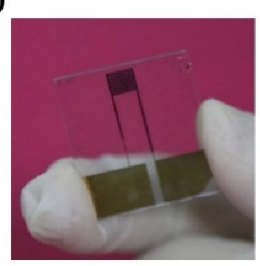

(b)

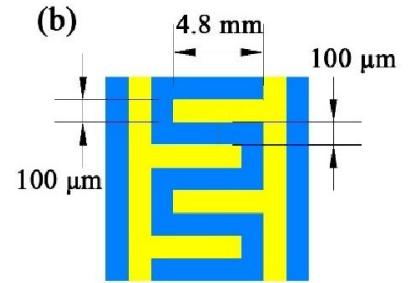

Figure 2 (a) Photograph of a real GBFC based on Au electrodes on a glass slide substrate, (b) Schematic diagram of the GBFC with 12 interdigital fingers.

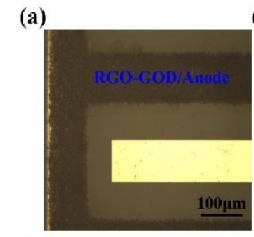

(d)
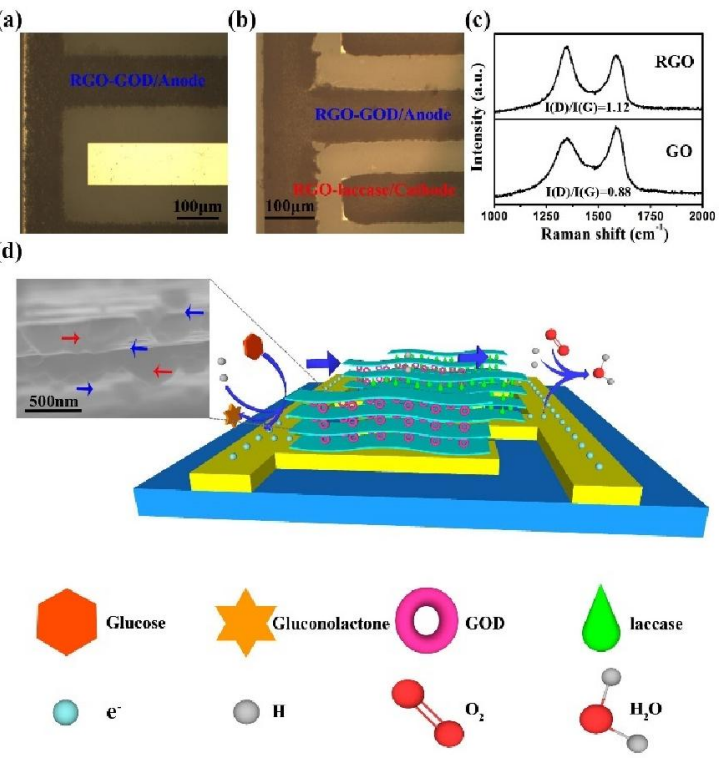

Figure 3 (a) Metallurgical microscope image of GBFC when there is only a bioanode being deposited. (b) Metallurgical microscope image of a full GBFC. (c) Raman spectra of the GO and RGO samples. (d) Schematic presentation of a full GBFC. Inset: FESEM image of RGO-enzyme hybrid, in which blue arrows point to the RGO layers while red arrows point to the enzymes. 
General Chemistry

clear boundary can be seen between each finger, thus preventing the short circuit resulting from the contact between the electrodes. Figure 3c showed Raman spectra of GO and RGO. $D$ band $\left(1345 \mathrm{~cm}^{-1}\right)$ represented the defects in $\mathrm{sp}^{3}$ hybridized carbon while $\mathrm{G}$ band $\left(1592 \mathrm{~cm}^{-1}\right)$ represented in-plane vibration in graphitic $\mathrm{sp}^{2}$ carbon structures, namely disorder and crystallinity, respectively. In this case, the intensity ratio between D-peek and G-peek increased from 0.88 to 1.12, which demonstrated obviously the transformation from GO to RGO. The schematic diagram of the full GBFC was presented in Figure $3 \mathrm{~d}$. In the bioanode, glucose was oxidized by GOD without redox mediator. The produced electrons flowed to the biocathode, where $\mathrm{O}_{2}$ was reduced to $\mathrm{H}_{2} \mathrm{O}$ through the catalyzation of laccase. Inset image was the morphology of RGO-enzyme hybrid, in which blue arrows pointed to the RGO layers while red arrows pointed to the enzymes. It's worth mentioning that this particular sandwich structure could not only increase the enzyme loading, but also speed up ion and electron transport.

The performances of bioanode and biocathode were tested in PBS containing appropriate concentration of glucose. In bioanode, glucose was catalyzed by GOD into glucono-1.5lactone to produce two electrons and protons [Eq. (1)], ${ }^{[2,23]}$

$D$-glucose GOD D-glucono-1.5-lactone $+2 \mathrm{H}^{+}+2 \mathrm{e}^{-}$

Different amount of GOD was added into the GO solution while the concentration of GO stayed the same before electrochemical deposition to optimize the bioanode performance. As can be seen in Figure $4 a$, the current density of bioanode strongly depended upon the amount of GOD incorporated in the deposition solution. The current density reached a maximum at about $30 \mathrm{mg}$ GOD and for GOD amount above $30 \mathrm{mg}$, the performance rapidly declined probably because a large amount of enzyme speeded up irreversibly precipitated agglomeration of $G O$ due to its strong $\pi-\pi$ interactions. The same question also existed in biocathode so that the amount of $30 \mathrm{mg}$ enzyme was applied to both electrodes.

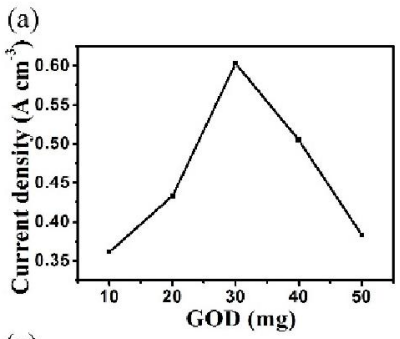

(b)
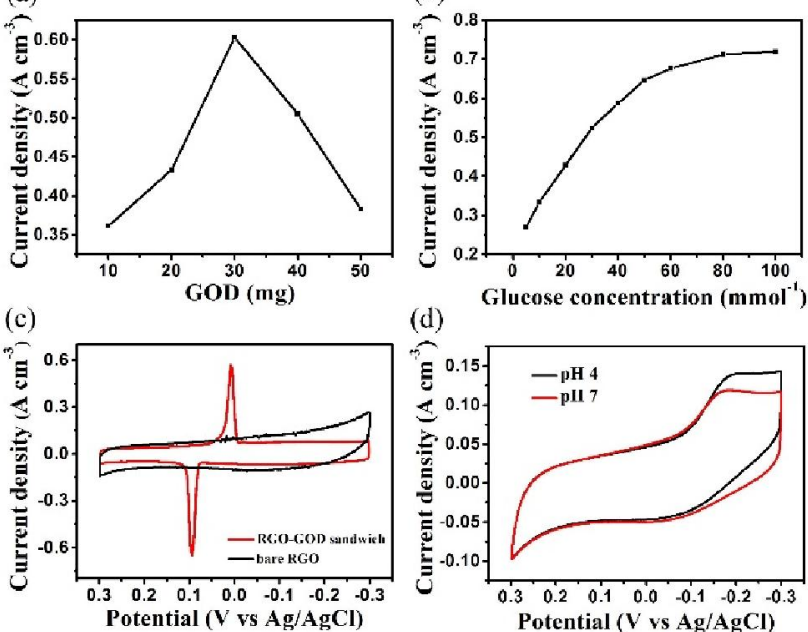

(d)

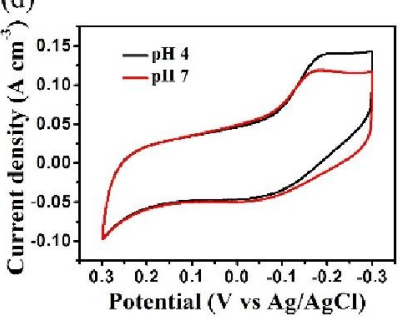

Figure 4 (a) Volumetric current density for the BFC anode as a function of GOD concentration. (b) Volumetric current density for the BFC anode as a function of glucose concentration. (c) CVs of bare RGO electrode (black) and RGO-GOD sandwich electrode (red) in $\mathrm{pH} 7$ electrolyte solution containing $50 \mathrm{mM}$ glucose. (d) CVs of RGO-laccase sandwich electrode in $\mathrm{pH} 4$ (black) air-saturated electrolyte solution and in $\mathrm{pH} 7$ (red) airsaturated electrolyte solution.
The concentration of the glucose also played an important role in bioanode performance. With the increase of glucose concentration from 5 to $100 \mathrm{mM}$, the anode current increased up to $0.72 \mathrm{~A} \cdot \mathrm{cm}^{-3}$ when operating in air-saturated PBS electrolyte, as shown in Figure $4 \mathrm{~b}$. It was noteworthy that the current density increased extremely fast when glucose concentration varies between 5 and $50 \mathrm{mM}$, corroborating the catalytic character of concentration towards the glucose oxidation. For higher glucose concentrations above $50 \mathrm{mM}$, the current density increased slowly and reached a constant value eventually. The resulting concentration-current density curve matched well with Michaelis-Menten saturation curve, which describing the relation in an enzyme reaction between reaction rate and substrate concentration. In this case, glucose was the only substrate of the enzyme catalytic reaction and the current, which came from the electronic transmission generated in the redox reaction, represented the reaction rate. The reaction speed indeed varied according to the change of the substrate concentration while the enzyme amount remained stable.

The electrochemical reactions of the RGO-GOD sandwich electrode were characterized by cyclic voltammetry (CV) as shown in Figure 4c. Noteworthy, the same test was performed with a similar bioanode without GOD to examine the possible contribution of the RGO to the catalytic oxidation of glucose. As expected, no redox peak was observed without GOD in the bare RGO electrode as shown in the black curve. In contrast, the red curve represented the electrochemical reaction of the RGO-GOD electrode and showed a pair of prominent peaks at 0 and $0.09 \mathrm{~V}$.

In the biocathode compartment, the RGO-laccase sandwich electrode received the electrons produced by bioanode oxidation and $\mathrm{O}_{2}$ was reduced to $\mathrm{H}_{2} \mathrm{O}$ through the catalyzation of laccase [Eq. (2)],

$$
\mathrm{O}_{2}+4 \mathrm{e}^{-}+4 \mathrm{H}^{+} \text {laccase } 2 \mathrm{H}_{2} \mathrm{O}
$$

To date, the majority of biocathodes used bilirubin oxidase (BOD) or laccace to reduce oxygen at the electrode surface. Among them, laccase was cheaper and more thoroughly studied so as to be utilized in this research. It was noteworthy that, as shown in Figure $4 \mathrm{~d}$, although laccase worked best at a pH of $\sim 4.5$ and may be inhibited by halide ions, such as $\mathrm{F}^{-}$and $\mathrm{Cl}^{-}$, the cathode current exhibited only a low decline when tested in PBS $(\mathrm{pH} 7)$ containing certain concentrations of chloride ion compared with the initial value. It highlighted the high stability of the immobilized laccase towards halide ions. ${ }^{[23,24]}$

A complete GBFC was constructed by the bioanode and biocathode as described. When operating in air-saturated PBS containing $50 \mathrm{mM}$ glucose at room temperature $\left(25{ }^{\circ} \mathrm{C}\right)$, generated an OCP of $+0.128 \mathrm{~V}$, which agreed with the performance gap between the individually measured OCP of bioelectrodes $(-0.08 \mathrm{~V}$ and $+0.06 \mathrm{~V}$ versus $\mathrm{Ag} / \mathrm{AgCl}$, respectively ). Figure 5 a showed the power density curve of the GBFC. The peak volumetric power density was about 14.77 $\mathrm{mW} \cdot \mathrm{cm}^{-3}$ when the operating voltage was about $0.091 \mathrm{~V}$. Usually, the cycle performance of enzyme-modified electrode can be key factors to confirm how long GBFC can maintain its power delivery. To study the stability of this GBFC, current retention was obtained by testing peak current of single electrode via cyclic voltammetry method for a RGO-enzyme hybrid electrode that contained GOD as the enzyme. As shown in Figure 5b, for 100 cycles, the peak current reached gradually maximum value and remained about the same until the end, which demonstrated clearly GBFC's excellent stability. Considering limitations, the performance of the GBFC would be clearly limited by cathodic bioelectrocatalytic reactions, which would definitely be responsible for a lowering of the voltage and 
the power density of GBFC because the enzyme active unit of laccase was much lower than GOD under the same weight. Further work was required to investigate the underlying mechanism of the electrochemical deposition process and the performance gap between bioanode and biocathode must be narrowed. (a)

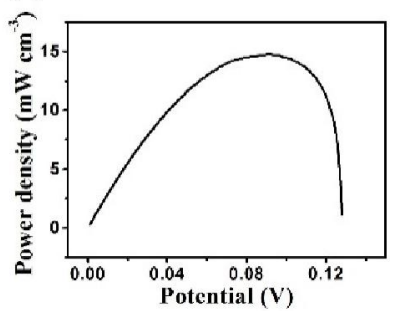

(b)

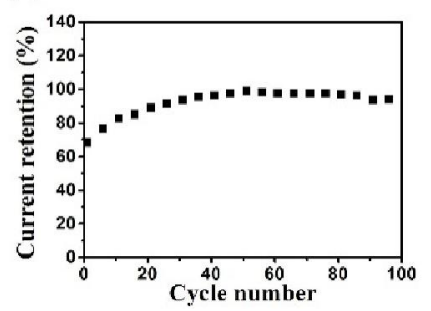

Figure 5 (a) Volumetric power density as a function of potential for the complete GBFC system at $50 \mathrm{mM}$ glucose concentration. (b) Current retention of the RGO-enzyme hybrid electrode for 100 cycles.

\section{Conclusions}

An on-chip, mediator- and membrane-less micro-GBFC based on layer-by-layer RGO-enzyme hybrids was demonstrated in this work. This mediator- and membrane-less design could narrow the gap between RGO and enzymes at the nanoscale level, thus realizing DET without the possible loss of enzyme activity. The obvious performance response of our developed GBFC confirmed the advantages of bioelectrode configuration that allowed enzymes to function in a three-dimensional conductive condition. Such unique layer-by-layer structure could effectively trap the enzymes and ensure high enzyme loading so that ion and electron transport could be sped up. This on-chip GBFC made implantation less unachievable and paved the road for developing implantable and selfcontained minimally biodevices, thus meeting the rapidly growing demands of modern society and solving the energy predicament.

\section{Acknowledgement}

We acknowledge the support from the National Natural Science Foundation of China (Nos. 51622208 and 21703149), the Natural Science Foundation of Jiangsu Province (No. BK20150338), and the Priority Academic Program Development of Jiangsu Higher Education Institutions (PAPD).

\section{References}

[1] Chen, Y.; Gai, P. P.; Xue, J. J.; Zhang, J. R.; Zhu, J. J. Biosens. Bioelectron. 2015, 74, 142.
[2] Meredith, M. T.; Minteer, S. D. Annu. Rev. Anal. Chem. 2012, 5, 157.

[3] Lang, Q. L.; Yin, L.; Shi, J. G.; Li, L.; Xia, L.; Liu, A. H. Biosens. Bioelectron. 2014, 51, 158

[4] Korani, A.; Salimi A.; Hadadzadeh, H. J. Power Sources 2015, 282, 586.

[5] Habrioux, A.; Napporn, T.; Servat, K.; Tingry, S.; Kokoh, K. B. Electrochim. Acta 2010, 55, 7701.

[6] Pankratov, D.; Sundberg, R.; Sotres, J.; Maximov, I.; Graczyk, M.; Suyatin, D. B.; Gonzalez-Arribas, E.; Lipkin, A.; Montelius, L.; Shleev, S. J. Power Sources 2015, 294, 501.

[7] Janegitz, B. C.; Pauliukaite, R.; Ghica, M. E.; Brett, C. M. A.; Fatibello-Filho, O. Sens. Actuators B 2011, 158, 411.

[8] Rotta, H. C. E. L.; Ciniciato, G. P. M. K.; González, E. R. Enzyme Microb. Technol. 2011, 48, 487.

[9] Gao, F.; Courjean, O.; Mano, N. Biosens. Bioelectron. 2009, 25, 356.

[10] Barrière, F.; Kavanagh, P.; Leech, D. Electrochim. Acta 2006, 51, 5187.

[11] Zebda, A.; Gondran, C.; Goff, A. L.; Holzinger, M.; Cinquin, P.; Cosnier, S. Nat. Commun. 2011, 2, 370

[12] Kwon, C. H.; Lee, S. H.; Choi, Y. B.; Lee, J. A.; Kim, S. H.; Kim, H H.; Spinks, G. M.; Wallace, G. G.; Lima, M. D.; Kozlov, M. E.; Baughman, R. H.; Kim, S. J. Nat. Commun. 2014, 5, 3928.

[13] Kwon, C. H.; Lee, J. A.; Choi, Y. B.; Kim, H. H.; Spinks, G. M.; Lima, M. D.; Baughman, R. H.; Kim, S. J. J. Power Sources 2015, 286 103.

[14] El-Kady, M. F.; Kaner, R. B. Nat. Commun. 2013, 4, 1475.

[15] El-Kady, M. F.; Strong, V.; Dubin, S.; Kaner, R. B. Science 2012 335,1326

[16] Zhu, A. Y.; Yi, F.; Reed, J. C.; Zhu, H.; Cubukcu, E. Nano Lett. 2014, $4,5641$.

[17] Zhang, W. J.; Li, X. J.; Zou, R.; Wu, H. Z.; Shi, H. Y.; Yu, S. S.; Liu, Y. Sci. Rep. 2015, 5, 1.

[18] Haddad, R.; Thery, J.; Gauthier-Manuel, B.; Elouarzaki, K.; Holzinger, M.; Goff, A. L.; Gautier, G.; Mansouri, J. E.; Martinent, A.; Cosnier, S. Electrochem. Commun. 2015, 54, 10.

[19] Si, W. P.; Yan, C. L.; Chen, Y.; Oswald, S.; Hana, L. Y.; Schmidt, O. G. Energy Environ. Sci. 2013, 6, 3218.

[20] Barton, S. C.; Gallaway, J.; Atanassov, P. Chem. Rev. 2004, 104, 4867.

[21] Hummers, W. S.; Offeman, R. E. J. Am. Chem. Soc. 1958, 80, 1339.

[22] Ramanavicius, A.; Genys, P.; Mikhailova, R.; Semashko, T.; Kausaite-Minkstimiene, A.; Morkvenaite-Vilkonciene, I.; Voronovic, J.; Ramanaviciene, A. Chem. Eng. J. 2015, 264, 165.

[23] Kima, R. E.; Honga, S. G.; Ha, S.; Kima, J. Enzyme Microb. Technol. 2014, 66, 35.

[24] Fokina, O.; Eipper, J.; Winandy, L.; Kerzenmacher, S.; Fischer, R. Bioresour. Technol. 2015, 175, 445. 